\title{
Real-time video image processing based on Kerr soliton crystal microcombs
}

\author{
Mengxi Tan, ${ }^{1}$ Xingyuan Xu, ${ }^{2}$ David J. Moss ${ }^{1 *}$ \\ ${ }^{1}$ Optical Science Centre, Swinburne University of Technology, Hawthorn, VIC 3122, Australia \\ ${ }^{2}$ Electro-Photonics Laboratory, Department of Electrical and Computer System Engineering, Monash University, Clayton, 3800 VIC, \\ Australia \\ *Corresponding author:dmoss@swin.edu.au
}

Advanced image processing will be crucial for emerging technologies such as autonomous driving, where the requirement to quickly recognize and classify objects under rapidly changing, poor visibility environments in real time will be needed. Photonic technologies will be key for nextgeneration signal and information processing, due to their wide bandwidths of 10's of Terahertz and versatility. Here, we demonstrate broadband real time analog image and video processing with an ultrahigh bandwidth photonic processor that is highly versatile and reconfigurable. It is capable of massively parallel processing over 10,000 video signals simultaneously in real time, performing key functions needed for object recognition, such as edge enhancement and detection. Our system, based on a soliton crystal Kerr optical micro-comb with a $49 \mathrm{GHz}$ spacing with $>\mathbf{9 0}$ wavelengths in the $\mathrm{C}$-band, is highly versatile, performing different functions without changing the physical hardware. These results highlight the potential for photonic processing based on Kerr microcombs for chip-scale fully programmable high-speed real time video processing for next generation technologies.

Keywords-Kerr frequency comb, Hilbert transform, integrated optics, all-optical signal processing, image processing, video image processing.

Image processing, the application of signal processing techniques to two-dimensional images such as photographs or video, is well known in the electronics world. [1] It is key for object recognition and classification, which are critical for automated decision making using machine learning for emerging technologies including robotic vision for self-driving cars [2], remote drones [3], automated in-vitro cellgrowth tracking for virus and cancer analysis [4], optical neural networks [5], ultrahigh speed imaging [6, 7] and many others. Many of these require real-time responses to massive real-world information. The volume of data for these applications, as well as the requirement for a real-time response, places extremely high demands on the processing bandwidths. Digital image processing of images using algorithms and digital computers, a branch of digital signal processing (DSP) [8], is well established but will be inadequate to meet these extreme demands due to limitations in processing speed (i.e., the electronic bandwidth) and the well-known von Neumann bottleneck [9].

Photonic RF techniques [10-15] have attracted significant interest over the past two decades due to their ability to provide ultra-high bandwidths, low transmission loss, and strong immunity to electromagnetic interference. They can perform signal processing functions in the optical domain, thus alleviating the bandwidth limitations imposed by analog-to-digital convertors [14] and digital electronics for DSP. Photonics has enabled significant emerging technologies, such as LIDAR for autonomous vehicles, that provides greatly enhanced performance under adverse environmental conditions, as compared with simple camera-based imaging [2].

Here, we demonstrate a photonic analog video image processor. It is based on a soliton crystal Kerr micro-comb source in an integrated micro-ring resonator (MRR) [5, 16-18]. We employ a reconfigurable photonic transversal structure to achieve a range of image processing functions for image edge enhancement, detection, motion blur and others, using up to 75 taps, or wavelengths. The device employs variable fractional order Hilbert transforms and differentiation, integration and bandpass filtering. The 
processing speed reaches 54 GigaBaud (pixels/s), capable of processing 10,000 video signals (1,200 high definition signals) in parallel. The experimental results agree well with theory, verifying our photonic processor as a new and competitive approach for analog image and video processing with a broad operation bandwidth, high scalability and reconfigurability, and potentially reduced cost and footprint.

\section{Soliton crystal micro-combs}

Integrated Kerr optical frequency combs based on micro-cavity resonators, or micro-combs, [16-39] have achieved significant breakthroughs in key emerging applications highlighted by range finding (LIDAR) [2], as well as many other areas including spectroscopy [40], communications [17, 41], optical neural networks [5], frequency synthesis [42], optical ranging [2, 43, 44], quantum sources [45, 46], metrology [47], and microwave photonics [15, 48-80].

Recently, a powerful category of micro-comb - soliton crystals - has attracted interest due to their crystallike profile in the angular domain in micro-ring resonators [17, 81-83]. They have underpinned breakthroughs in microwave and RF photonics [15, 50], ultrahigh bandwidth communications [17] and optical neuromorphic processing [5]. Their robustness is central to providing stable micro-combs without the need for complex feedback systems. They can be generated simply and deterministically, driven by a mode crossing-induced background wave interacting via the Kerr nonlinearity at high intra-cavity powers. Because the intra-cavity energy of the soliton crystal state is similar to the chaotic state from which they originate, there is no significant change in intracavity energy when they are generated. Hence, there is very little self-induced thermal detuning shift, known as the characteristic 'soliton step', that requires complex tuning methods [17-21] to compensate for in the case of single solitons. This has the important result that soliton crystals can be generated through manual adiabatic pump wavelength sweeping - a simple and reliable initiation process that also results in a much higher energy efficiency (ratio of optical power in the comb lines relative to the pump power) [17].

The MRR used to generate soliton crystal micro-combs was fabricated in a CMOS compatible doped silica glass platform [16, 17] with a $Q$ factor of $\sim 1.5$ million, radius $\sim 592 \mu \mathrm{m}$, and FSR $\sim 0.393 \mathrm{~nm}(48.9 \mathrm{GHz})$. This is a very low FSR spacing for an integrated micro-comb source and is critical to this work since it resulted in a large number of wavelengths over the $\mathrm{C}$-band. The chip was coupled with a fibre array, featuring a fibre-chip coupling loss of only $0.5 \mathrm{~dB}$ /facet with integrated mode converters. The crosssection of the waveguide was $3 \mu \mathrm{m} \times 2 \mu \mathrm{m}$, yielding anomalous dispersion in the $\mathrm{C}$ band as well as the mode crossing at $\sim 1552 \mathrm{~nm}$.

To generate the micro-combs, a CW pump laser was amplified to $30.5 \mathrm{dBm}$ and the wavelength manually swept from blue to red. When the detuning between pump wavelength and MRR's cold resonance was small enough, the intra-cavity power (Fig. 1 (b)) reached a threshold and modulation instability (MI) driven oscillation resulted. Primary combs (Fig. 1 (ii) (iii)) were generated with a spacing determined by the MI gain peak, a function of the intra-cavity power and dispersion. As the detuning was changed further, a second jump in the intra-cavity power was observed, where distinctive 'fingerprint' optical spectra (Fig. 1 (iv)) appeared from the soliton crystals [17, 81-83]. Their spectral shape arises from spectral interference between the tightly packed solitons circulating along the ring cavity. We present theoretical results that support the generation of soliton crystal micro-comb (Supplementary Movie S1). The power fluctuations of the micro-comb were measured over 140 hours (5 days), with the optical spectrum captured every 15 minutes (Fig. 1 (c)), indicating that the micro-comb source is a stable multiwavelength source for the analog video processor.

\section{Analog image processing}

Signal processing is critical for image and video analysis [84-90] to perform functions such as object identification, that include integral and fractional differentiators for edge detection [85-87], fractional 
Hilbert transformers for edge enhancement [88], integrators and bandpass filters for motion blur [89]. Motion blur is the apparent streaking of moving objects in a photograph or a sequence of frames, and arises when the image being recorded changes during the recording of a single exposure, due to rapid movement or long exposure [89,90]. Many of these functions are also used in RF applications such as radar systems, signal sideband modulators, measurement systems, signal sampling, and communications $[14,85]$. They will be critical for emerging applications such as lidar for autonomous vehicles [2].

Figure 2 illustrates the conceptual diagram for the photonic analog image and video processor. First, the input frame was flattened into a vector $x$ and encoded as the intensity of temporal symbols in a serial electrical waveform at a sampling rate of 54 GBaud with a nominal resolution of 8 bits (see Supplementary for a discussion of the effective number of bits (ENOBs). The impulse response of the image processor is represented by $N(=75)$ tap weights $(h)$ that encode the optical power of the microcomb lines via spectral shaping with a WaveShaper.

The input waveform $x$ was multi-cast onto the $N$ shaped comb lines via electro-optical modulation, yielding $N$ replicas weighted by the tap weights $h$. The waveform was then transmitted through $3.96 \mathrm{~km}$ length of fibre to generate a relative delay between wavelengths. Finally, the replicas were summed by photodetection, given by

$$
F(\omega)=\sum_{n=0}^{N-1} h(n) e^{-j \omega n T}
$$

where $\omega$ is the RF angular frequency, $T$ is the time delay between adjacent taps, and $h(n)$ is the tap coefficient of the $\mathrm{n}^{\text {th }}$ tap, which is the discrete impulse response of the transfer function $H(\omega)$ of the signal processor. The discrete impulse response $h(n)$ can be calculated by performing the inverse Fourier transform of the transfer function $H(\omega)$ of the signal processor $[15,50]$. The output waveform $y$ was then combined before reconstruction.

For a multi-wavelength optical carrier transmitted over a dispersive medium, the relative time delay between adjacent wavelengths is

$$
T=D \times L \times \Delta \lambda
$$

where $D$ denotes the dispersion coefficient, $L$ denotes the length of the dispersive medium, and $\Delta \lambda$ represents the wavelength spacing of the soliton crystal micro-comb, as shown in Fig. 1 (a). Figure 3 illustrates the experimental set-up, which consists of two parts - the comb generation and flattening module, and the transversal structure. The soliton crystal micro-comb spectrum was pre-flattened from the initial scallop shaped spectrum by the first WaveShaper (Finisar 4000S). The flattened comb lines were then modulated by the serial electrical waveform, effectively multicasting the electrical signal onto all wavelengths. The input electronic signal then was transmitted through $3.96 \mathrm{~km}$ of standard single mode fibre with a dispersion $\sim 17 \mathrm{ps} / \mathrm{nm} / \mathrm{km}$, to yield the progressive delay taps, with a relative inter-tap time delay between adjacent wavelengths of $T=27.08 \mathrm{ps}$. The second WaveShaper then equalized and weighted the power of the comb lines according to the designed tap weights. Finally, the weighted and delayed taps were combined and converted back into the electronic domain via high speed photodetection (Finisar BPDV2150R). By tailoring the comb lines' power according to the tap coefficients, arbitrary phase shifts for the Hilbert transformer and fractional orders of the differentiator could be achieved.

Fig. 1 (a) shows the relationship between the wavelength spacing of the comb, the total delay of the fibre, and the resulting RF FSR, or essentially the Nyquist zone. The RF operation bandwidth of the analog image processor is half of the free $\mathrm{RF}$ spectral range $\left(F S R_{\mathrm{RF}}\right)$, given by $F S R_{\mathrm{RF}}=1 / T$, yielding $B W_{R F} \sim 18$ GHz. Note that although the use of fibre resulted in a significant signal latency, it did not affect the device throughput speed. Further, this latency can be virtually eliminated by using any one of a number of compact dispersive components such as fibre Bragg gratings (FBGs) [91] or tunable dispersion compensators [92].

Figure 4 illustrates the simulated and experimental results of the shaped comb spectra, including the temporal impulse response, frequency response and processed image for a differentiator with a fractional 
order of $0.5,0.75$, and 1 , an integrator with numbers of 15,45 , and 75 taps, as well as a Hilbert transformer with an operation bandwidth of 12,18 , and $38 \mathrm{GHz}$. Fractional differentiation performs edge detection, while Hilbert transforms perform edge enhancement or 'sharpening'. Both of these apply to both static images as well as frames of video signals. Integration and bandpass filters address the issue of motion blur (Fig. 4 (i)). The transmission response (Fig. 4 (ii)) was characterized by a vector network analyser (Agilent MS4644B). By varying the comb spacing as well as the fibre length, the operation bandwidth of the Hilbert transformer with a $90^{\circ}$ phase shift could be adjusted from 12-38 GHz. Fig. 4 (iii) shows the simulated and measured processed images for different functions. The original high definition (HD) image was captured by a Nikon camera (D5600) with a resolution of $1080 \times 1620$ pixels. The processed images after 0.5, 0.75, and first-order differentiation were shown in Fig. 4 (a-iii) (b-iii) (c-iii), respectively, which indicate that the edge of the image was successfully detected. Fig. 4 (d -iii) (e-iii) (f-iii) show the processed images after integration with 15,45 , and 75 taps, respectively, where we see that the blur intensity increases with the number of taps. The processed images after the Hilbert transformation for operation bandwidths of 12-38 GHz are shown in Fig. 4 (g-iii) (h-iii) (I-iii), respectively.

\section{Real time analog video processing}

To process videos in real time we use a combination of fractional differentiator (order $=0.5$ ), an integrator with 75 taps, and Hilbert transformer with a bandwidth of $18 \mathrm{GHz}$. Fig. 5 (a) shows the generated waveform together with 5 frames of the original video at a frame rate of 30 frames per second. The video had a resolution of $568 \times 320$ pixels and was captured by a Drone Quadcopter UAV with Optical Zoom camera (DJL Mavic Air 2 Zoom). The video after differentiation and Hilbert transformation is shown in Fig. 5 (b). Fig. 5 (c) and (d). The input and processed HD videos as well as the waveforms are seen here

Movie S2 - video process.mp4 (Command Line)

For demonstrating a reconfigurable operation bandwidth, we focus on the Hilbert transformer, showing a variable range of $12-38 \mathrm{GHz}$ with a phase shift of $90^{\circ}$, achieved by varying the length of fibre $(1.838 \mathrm{~km}$ vs $3.96 \mathrm{~km}$ ) as well as by varying the comb spacing (with a 2-FSR and 3-FSR comb spacing) Fig. 4 (g-ii) (hii) (I-ii). Note that tunable dispersion compensators [92] can be employed to avoid changing the hardware to vary the bandwidths.

\section{Discussion}

To quantitatively evaluate the performance of our processor, we evaluated the edge detection using a ground truth for quantitative and qualitative comparisons [90]. We use 3 BSD (Berkeley Segmentation Database) images and respective ground truths for our evaluation, with performance parameters including PR (Performance Ratio) and F-Measure (higher values of these parameters reflect a better edge detection). Fig. 6 shows the simulated and experimental edge detection results of 3 BSD images with different processing approaches (Sobel and different fractional orders of differentiation). The performance and comparative results are shown in Table 1. The fractional differentiation results are compared with Sobel's algorithm using ground truth of respective images, where we see that our experimental results for PR and F-Measures are better than Sobel's approach.

The maximum speed of our system is 54 GBaud , or Gigapixels/s, which, with a video resolution of $568 \times$ 320 yields 181,760 pixels at a frame rate of $30 \mathrm{~Hz}$, resulting in a processing capacity of $54 \times 10^{9}$ / $5,452,800=10,000$ video signals simultaneously in real time. For high definition video $(720 \times 1280=$ 921,600 pixels) at $50 \mathrm{~Hz}$, this equates to about 1,200 video signals in parallel. This can be increased even further by increasing the channel spacing (using wider spaced FSRs) or by other methods such as using the telecommunications L-band in parallel.

Although the experiments reported here included benchtop components, such as the commercially available WaveShaper, there is potential for much higher levels of integration, even for full monolithic integration. The core component of our system, the microcomb, is already integrated. Further, all of the other components have been demonstrated in integrated form, including integrated InP spectral shapers 
[93], high-speed integrated lithium niobite modulators [94], integrated dispersive elements [91], and photodetectors [95]. Finally, low power - consumption [96] and highly efficient laser cavity-soliton [97] Kerr combs have recently been demonstrated, that would greatly reduce the energy requirements.

\section{Conclusion}

In conclusion, we demonstrate the first photonic-based analog image and video processor operating with functions designed for edge enhancement, detection, and motion blur, that perform fractional Hilbert transforms with tunable phase shift from $15^{\circ}$ to $75^{\circ}$, fractional differentiation with tunable order from 0.1 to 0.9 , non-fractional (integral) signal processing with a $90^{\circ}$ phase shift Hilbert transform, $1^{\text {st }}$ - order differentiation, and integration. The system is capable of processing 10,000 video images or 1,200 high definition video signals simultaneously in real time. The experimental results agree well with theory, verifying that the photonic-based analog image and video processor has a broad operation bandwidth, high reconfigurability, with potentially reduced cost and footprint.

\section{References}

[1] Petrou M, Bosdogianni P, Image processing: the fundamentals. John Wiley (1999)

[2] J. Riemensberger, A. Lukashchuk, M. Karpov, W. Weng, E. Lucas, J. Liu, T. J. Kippenberg, "Massively parallel coherent laser ranging using a soliton microcomb", Nature $\underline{\mathbf{5 8 1}}$ (7807), 164-170 (2020).

[3] Hodge, V.J., Hawkins, R. \& Alexander, R. Deep reinforcement learning for drone navigation using sensor data. Neural Comput \& Applic 33, 2015-2033 (2021).

[4] Fusciello, M., Fontana, F., Tähtinen, S. et al. Artificially cloaked viral nano-vaccine for cancer immunotherapy. Nat Commun 10, 5747 (2019).

[5] X. Xu, M. Tan, B. Corcoran, J. Wu, A. Boes, T. G. Nguyen, S. T. Chu, B. E. Little, D. G. Hicks, R. Morandotti, A. Mitchell, and D. J. Moss,., "11 TOPS photonic convolutional accelerator for optical neural networks", Nature 589, 44-51 (2021).

[6] P. Wang, J. Liang, \& L.V. Wang, "Single-shot ultrafast imaging attaining 70 trillion frames per second", Nature Communications (2020). https://doi.org/10.1038/s41467-020-15745-4

[7] L. Gao, J. Liang, C. Li, L.V. Wang, "Single-shot compressed ultrafast photography at one hundred billion frames per second", Nature $\underline{\mathbf{5 1 6}}$ (7529), 74-77 (2014).

[8] Gonzalez, Rafael, Digital image processing. New York, NY: Pearson. ISBN 978-0-13-335672-4. OCLC 966609831, (2018).

[9] Backus, John, "Can Programming Be Liberated from the von Neumann Style? A Functional Style and Its Algebra of Programs" (PDF). Communications of the ACM. Vol. 21, No. 8: 613-641. Retrieved September 19, 2020 - via Karl Crary, School of Computer Science, Carnegie Mellon University, (August 1978).

[10] J. Capmany, and D. Novak, "Microwave photonics combines two worlds," Nat. Photonics 1, 319-330 (2007).

[11] J. P. Yao, “Microwave photonics," Journal of Lightwave Technol. 27, 314-335 (2009).

[12] D. Marpaung, J. Yao, and J. Capmany, "Integrated microwave photonics," Nature Photonics 13 (2019).

[13] W. Liu, et al., "A Fully Reconfigurable Photonic Integrated Signal Processor," Nature Photonics 10 (3), 190-196 (2016).

[14] J. Capmany, J. Mora, I. Gasulla, J. Sancho, J. Lloret, and S. Sales, "Microwave Photonic Signal Processing," Journal of Lightwave Technol. 31, (4), 571-586 (2013).

[15] J. Wu et al., "RF photonics: An optical micro-combs' perspective", IEEE Journal of Selected Topics in Quantum Electronics 24, (4), 1-20, Article: 6101020 (2018).

[16] D. J. Moss, R. Morandotti, A. L. Gaeta, M. Lipson, New CMOS-compatible platforms based on silicon nitride and Hydex for nonlinear optics. Nature photonics 7, 597 (2013). 
[17] B. Corcoran et al., "Ultra-dense optical data transmission over standard fiber with a single chip source", Nature Communications 11, 2568 (2020).

[18] Pasquazi, A. et al. Micro-combs: A novel generation of optical sources. Phys. Rep. 729, 1-81 (2017).

[19] T. J. Kippenberg, R. Holzwarth, S. A. Diddams, Microresonator-based optical frequency combs. Science 332, 555-559 (2011).

[20] T.J. Kippenberg, A.L. Gaeta, M. Lipson, M.L. Gorodetsky, "Dissipative Kerr solitons in optical microresonators", Science 361, (6402) eaan8083 (2018).

[21] A.L. Gaeta, M. Lipson, T.J. Kippenberg, "Photonic-chip-based frequency combs", Nature Photonics, vol. 13 (3), 158-169 (2019).

[22] L. Razzari et al., "CMOS-compatible integrated optical hyper-parametric oscillator,” Nat. Photonics, vol. 4, no. 1, pp. 41-45, Jan. 2010.

[23] P. Roztocki, M. Kues, C. Reimer, B. Wetzel, S. Sciara, Y. Zhang, A. Cino, B. E. Little, S. T. Chu, D. J. Moss, and R. Morandotti, "Practical system for the generation of pulsed quantum frequency combs," Optics Express, vol. 25, no. 16, pp. 18940-18949, 2017.

[24] M. Kues, C. Reimer, A. Weiner, J. Lukens, W. Munro, D. J. Moss, and R. Morandotti, “Quantum Optical Micro-combs", Nature Photonics, vol. 13, no.3, pp. 170-179, 2019.

[25] C. Reimer, et al.,"High-dimensional one-way quantum processing implemented on d-level cluster states", Nature Physics, vol. 15, no.2, pp. 148-153, 2019.

[26] A. Pasquazi, et al., "Sub-picosecond phase-sensitive optical pulse characterization on a chip", Nature Photonics, vol. 5, no. 10, pp. 618-623 (2011). DOI: 10.1038/nphoton.2011.199.

[27] L. D. Lauro, J. Li, D. J. Moss, R. Morandotti, S. T. Chu, M. Peccianti, and A. Pasquazi, "Parametric control of thermal self-pulsation in micro-cavities," Opt. Lett. vol. 42, no. 17, pp. 3407-3410, Aug. 2017.

[28] H. Bao et al., "Type-II micro-comb generation in a filter-driven four wave mixing laser," Photonics Research, vol. 6, no. 5, pp. B67-B73 (2018).

[29] M. Ferrera et al., "Low-power continuous-wave nonlinear optics in doped silica glass integrated waveguide structures," Nat. Photonics, vol. 2, no. 12, pp. 737-740, Nov. 2008.

[30] D. Duchesne, M. Peccianti, M. R. E. Lamont, et al., "Supercontinuum generation in a high index doped silica glass spiral waveguide," Optics Express, vol. 18, no, 2, pp. 923-930, 2010.

[31] M. Ferrera, et al., "On-chip CMOS-compatible all-optical integrator”, Nature Communications, vol. 1, Article 29, 2010. DOI:10.1038/ncomms1028

[32] H.Bao, et al., "Turing patterns in a fibre laser with a nested micro-resonator: robust and controllable microcomb generation", Physical Review Research, vol. 2, $023395 \quad$ (2020). DOI: 10.1103/PhysRevResearch.00.003000.

[33] A. Pasquazi, Y. Park, J. Azana, et al., "Efficient wavelength conversion and net parametric gain via Four Wave Mixing in a high index doped silica waveguide," Optics Express, vol. 18, no. 8, pp. 7634-7641, 2010 .

[34] M. Peccianti, M. Ferrera, L. Razzari, et al., "Subpicosecond optical pulse compression via an integrated nonlinear chirper," Optics Express, vol. 18, no. 8, pp. 7625-7633, 2010.

[35] D. Duchesne, M. Ferrera, L. Razzari, et al., "Efficient self-phase modulation in low loss, high index doped silica glass integrated waveguides," Optics Express, vol. 17, no. 3, pp. 1865-1870, 2009.

[36] M. Peccianti, et al., "Demonstration of an ultrafast nonlinear microcavity modelocked laser", Nature Communications, vol. 3, pp. 765, 2012. DOI:10.1038/ncomms 1762

[37] M. Kues, et al., "Passively modelocked laser with an ultra-narrow spectral width", Nature Photonics, vol. 11, no. 3, pp. 159, 2017. DOI:10.1038/nphoton.2016.271

[38] A. Pasquazi, L. Caspani, M. Peccianti, et al., "Self-locked optical parametric oscillation in a CMOS compatible microring resonator: a route to robust optical frequency comb generation on a chip," Optics Express, vol. 21, no. 11, pp. 13333-13341, 2013. 
[39] C. Reimer, et al., "Cross-polarized photon-pair generation and bi-chromatically pumped optical parametric oscillation on a chip", Nature Communications, vol. 6, Article 8236, 2015. DOI: 10.1038/ncomms9236

[40] Suh, M.-G., Yang, Q.-F., Yang, K. Y., Yi, X. \& Vahala, K. J. Microresonator soliton dual-comb spectroscopy. Science 354, 600-603 (2016).

[41] Marin-Palomo, P. et al. Microresonator-based solitons for massively parallel coherent optical communications. Nature 546, 274-279 (2017).

[42] Spencer, D. T. et al. An Optical-Frequency Synthesizer Using Integrated Photonics, Nature 557, 81-85 (2018).

[43] Trocha, P. et al. Ultrafast optical ranging using microresonator soliton frequency combs. Science 359, 887 891 (2018).

[44] Suh, M.-S. \& Vahala, K. J. Soliton microcomb range measurement. Science 359, 884-887 (2018)

[45] Kues, M. et al. On-chip generation of high-dimensional entangled quantum states and their coherent control. Nature 546, 622-626 (2017).

[46] Reimer, C. et al. Generation of multiphoton entangled quantum states by means of integrated frequency combs. Science 351, 1176-1180 (2016).

[47] Brasch, V. et al. Photonic chip-based optical frequency comb using soliton Cherenkov radiation. Science 351, 357-360 (2016).

[48] Del'Haye, P. et al. Phase-coherent microwave-to-optical link with a self-referenced microcomb. Nat. Photonics 10, 516-520 (2016).

[49] Liang, W., Eliyahu, D., Ilchenko, V. S., Savchenkov, A. A., Matsko, A. B., Seidel, D., \& Maleki, L. High spectral purity Kerr frequency comb radio frequency photonic oscillator. Nat. Commun. 6, 7957 (2015).

[50] X. Xu, M. Tan, J. Wu, R. Morandotti, A. Mitchell and D. J. Moss, Microcomb-based photonic RF signal processing. IEEE Photonics Technology Letters 31, 1854-1857 (2019).

[51] X. Xu, J. Wu, M. Shoeiby, T. G. Nguyen, S. T. Chu, B. E. Little, R. Morandotti, A. Mitchell, and D. J. Moss, "Reconfigurable broadband microwave photonic intensity differentiator based on an integrated optical frequency comb source," APL Photonics, vol. 2, no. 9, 096104 (2017).

[52] T. G. Nguyen et al., "Integrated frequency comb source-based Hilbert transformer for wideband microwave photonic phase analysis,” Opt. Express, vol. 23, no. 17, pp. 22087-22097 (2015).

[53] X. Xu, et al., "Advanced RF and microwave functions based on an integrated optical frequency comb source," Opt. Express, vol. 26, no. 3, pp. 2569-2583 (2018).

[54] X. Xu, et al., "Broadband RF channelizer based on an integrated optical frequency Kerr comb source," Journal of Lightwave Technology, vol. 36, no. 19, pp. 4519-4526 (2018).

[55] X. Xu, et al., "Continuously tunable orthogonally polarized RF optical single sideband generator based on micro-ring resonators," Journal of Optics, vol. 20, no. 11, 115701 (2018).

[56] X. Xu, et al., "Orthogonally polarized RF optical single sideband generation and dual-channel equalization based on an integrated microring resonator," Journal of Lightwave Technology, vol. 36, no. 20, pp. 4808-4818 (2018).

[57] X. Xu, et al., "Photonic microwave true time delays for phased array antennas using a $49 \mathrm{GHz}$ FSR integrated optical micro-comb source," Photonics Res, vol. 6, no. 5, pp. B30-B36 (2018).

[58] X. Xu, et al., "Advanced adaptive photonic RF filters with 80 taps based on an integrated optical micro-comb source," Journal of Lightwave Technology, vol. 37, no. 4, pp. 1288-1295 (2019).

[59] X. Xu et al., Broadband microwave frequency conversion based on an integrated optical micro-comb source", Journal of Lightwave Technology, vol. 38 no. 2, pp. 332-338 (2020).

[60] M. Tan et al., "Photonic RF and microwave filters based on 49GHz and 200GHz Kerr microcombs", Optics Comm. vol. 465, Article: 125563, Feb. 22 (2020).

[61] X. Xu et al., "Broadband photonic RF channelizer with 90 channels based on a soliton crystal microcomb", Journal of Lightwave Technology, Vol. 38, no. 18, pp.5116 - 5121 (2020). doi: 10.1109/JLT.2020.2997699. 
[62] X. Xu et al., "Photonic RF and microwave integrator with soliton crystal microcombs", IEEE Transactions on Circuits and Systems II: Express Briefs, Vol. 67 (12) 3582-3586 (2020). DOI:10.1109/TCSII.2020.2995682.

[63] X. Xu et al., "Photonic RF phase-encoded signal generation with a microcomb source", Journal of Lightwave Technology, vol. 38, no. 7, pp. 1722-1727 (2020).

[64] X. Xu et al., "High performance RF filters via bandwidth scaling with Kerr micro-combs," APL Photonics, vol. 4, no. 2, pp. 026102 (2019).

[65] M. Tan et al., "Microwave and RF photonic fractional Hilbert transformer based on a 50 GHz Kerr microcomb", Journal of Lightwave Technology, vol. 37, no. 24, pp. 6097 - 6104 (2019).

[66] M. Tan et al., "RF and microwave fractional differentiator based on photonics", IEEE Transactions on Circuits and Systems: Express Briefs, Vol. 67, No. 11, pp. 2767 - 2771 (2020). DOI:10.1109/TCSII.2020.2965158.

[67] M. Tan et al., "Photonic RF arbitrary waveform generator based on a soliton crystal micro-comb source", Journal of Lightwave Technology, Vol. 38, No. 22, pp. 6221-6226, Oct 22 (2020). DOI: 10.1109/JLT.2020.3009655.

[68] M. Tan, X. Xu, J. Wu, R. Morandotti, A. Mitchell, and D. J. Moss, "RF and microwave high bandwidth signal processing based on Kerr Micro-combs", Advances in Physics X, VOL. 6, NO. 1, 1838946 (2020). DOI:10.1080/23746149.2020.1838946.

[69] Mengxi Tan, Xingyuan Xu, Jiayang Wu, Thach G. Nguyen, Sai T. Chu, Brent E. Little, Roberto Morandotti, Arnan Mitchell, and David J. Moss, "Photonic Radio Frequency Channelizers based on Kerr Micro-combs and Integrated Micro-ring Resonators”, (2020). JOSarXiv.202010.0002.

[70] Mengxi Tan, Xingyuan Xu, David Moss “Tunable Broadband RF Photonic Fractional Hilbert Transformer Based on a Soliton Crystal Microcomb”, Preprints, DOI: 10.20944/preprints202104.0162.v1

[71] Mengxi Tan, X. Xu, J. Wu, T. G. Nguyen, S. T. Chu, B. E. Little, R. Morandotti, A. Mitchell, and David J. Moss, "Orthogonally polarized Photonic Radio Frequency single sideband generation with integrated microring resonators", Journal of Semiconductors $\underline{\mathbf{4 2}}$ (4), 041305 (2021). $\quad$ DOI: 10.1088/16744926/42/4/041305.

[72] Mengxi Tan, X. Xu, J. Wu, T. G. Nguyen, S. T. Chu, B. E. Little, R. Morandotti, A. Mitchell, and David J. Moss, "Photonic Radio Frequency Channelizers based on Kerr Optical Micro-combs", Journal of Semiconductors $\underline{42}$ (4), 041302 (2021). (ISSN 1674-4926).

DOI: $10.1088 / 1674-4926 / 42 / 4 / 041302$.

[73] H.Bao, L.Olivieri, M.Rowley, S.T. Chu, B.E. Little, R.Morandotti, D.J. Moss, J.S.T. Gongora, M.Peccianti and A.Pasquazi, "Laser Cavity Solitons and Turing Patterns in Microresonator Filtered Lasers: properties and perspectives”, Paper No. LA203-5, Paper No. 11672-5, SPIE LASE, SPIE Photonics West, San Francisco CA March 6-11 (2021). DOI: $\underline{10.1117 / 12.2576645}$

[74] Mengxi Tan, X. Xu, J. Wu, A. Boes, T. G. Nguyen, S. T. Chu, B. E. Little, R. Morandotti, A. Mitchell, and David J. Moss, "Advanced microwave signal generation and processing with soliton crystal microcombs", or "Photonic convolutional accelerator and neural network in the Tera-OPs regime based on Kerr microcombs", Paper No. 11689-38, PW21O-OE201-67, Integrated Optics: Devices, Materials, and Technologies XXV, SPIE Photonics West, San Francisco CA March 6-11 (2021). DOI: 10.1117/12.2584017

[75] Mengxi Tan, Bill Corcoran, Xingyuan Xu, Andrew Boes, Jiayang Wu, Thach Nguyen, Sai T. Chu, Brent E. Little, Roberto Morandotti, Arnan Mitchell, and David J. Moss, "Optical data transmission at 40 Terabits/s with a Kerr soliton crystal microcomb”, Paper No.11713-8, PW21O-OE803-23, Next-Generation Optical Communication: Components, Sub-Systems, and Systems X, SPIE Photonics West, San Francisco CA March 6-11 (2021). DOI:10.1117/12.2584014

[76] Mengxi Tan, X. Xu, J. Wu, A. Boes, T. G. Nguyen, S. T. Chu, B. E. Little, R. Morandotti, A. Mitchell, and David J. Moss, "RF and microwave photonic, fractional differentiation, integration, and Hilbert transforms based on Kerr micro-combs", Paper No. 11713-16, PW21O-OE803-24, Next-Generation Optical Communication: Components, Sub-Systems, and Systems X, SPIE Photonics West, San Francisco CA March 6-11 (2021). DOI:10.1117/12.2584018

[77] Mengxi Tan, X. Xu, J. Wu, A. Boes, T. G. Nguyen, S. T. Chu, B. E. Little, R. Morandotti, A. Mitchell, and 
David J. Moss, "Broadband photonic RF channelizer with 90 channels based on a soliton crystal microcomb", or "Photonic microwave and RF channelizers based on Kerr micro-combs", Paper No. 11685-22, PW21OOE106-49, Terahertz, RF, Millimeter, and Submillimeter-Wave Technology and Applications XIV, SPIE Photonics West, San Francisco CA March 6-11 (2021). DOI:10.1117/12.2584015

[78] X. Xu, M. Tan, J. Wu, S. T. Chu, B. E. Little, R. Morandotti, A. Mitchell, B. Corcoran, D. Hicks, and D. J. Moss, "Photonic perceptron based on a Kerr microcomb for scalable high speed optical neural networks", IEEE Topical Meeting on Microwave Photonics (MPW), pp. 220-224,Matsue, Japan, November 24-26, 2020. Electronic ISBN:978-4-88552-331-1.

DOI: $10.23919 / \mathrm{MWP} 48676.2020 .9314409$

[79] Mengxi Tan, Bill Corcoran, Xingyuan Xu, Andrew Boes, Jiayang Wu, Thach Nguyen, S.T. Chu, B. E. Little, Roberto Morandotti, Arnan Mitchell, and David J. Moss, "Ultra-high bandwidth optical data transmission with a microcomb", IEEE Topical Meeting on Microwave Photonics (MPW), pp. 78-82.Virtual Conf., Matsue, Japan, November 24-26, 2020. Electronic ISBN:978-4-88552-331-1.

\section{DOI: $10.23919 / \mathrm{MWP} 48676.2020 .9314476$}

[80] Mengxi Tan, Xingyuan Xu, Jiayang Wu, Thach G. Nguyen, Sai T. Chu, Brent E. Littl, Arnan Mitchell, Roberto Morandotti, and David J. Moss, "Orthogonally polarized RF optical single sideband generation with integrated ring resonators”, (2020). JOSarXiv.202010.0001.

[81] Cole, D. C., Lamb, E. S., Del'Haye, P., Diddams, S. A. \& Papp, S. B. Soliton crystals in Kerr resonators. Nat. Photonics 11, 671-676 (2017).

[82] Wang, W., Lu, Z., Zhang, Z., Chu, S.T., Little, B.E., Wang, L., Xie, P., Liu, M., Yang, Q., Wang, L., Zhao, J., Wang, G., Sun, Q., Liu, Y., Wang, Y. \& Zhao, W., Robust soliton crystals in a thermally controlled microresonator, Opt. Lett. 43, 2002 (2018).

[83] M. Karpov, M.H.P. Pfeiffer, H. Guo, W. Weng, J. Liu, T.J. Kippenberg, "Dynamics of soliton crystals in optical microresonators", Nature Physics 15, (10), 1071-1077 (2019).

[84] Umbaugh, Scott E, Digital image processing and analysis: human and computer vision applications with CVIPtools (2nd ed.). Boca Raton, FL: CRC Press. ISBN 978-1-4398-0205-2, (2010).

[85] T. Yang, J. Dong, L. Liu, S. Liao, S. Tan, L. Shi, D. Gao, and X. Zhang, "Experimental observation of optical differentiation and optical Hilbert transformation using a single SOI microdisk chip," Scientific Reports 4, 3960 (2014).

[86] B. Mathieu, P. Melchior, A. Oustaloup, Ch. Ceyral, "Fractional differentiation for edge detection," Signal Processing 83, 2421-2432 (2003).

[87] J. C. Bancroft, and H. D. Geiger, “Analysis and design of filters for differentiation,” CREWES Research Report 9, 21 (1997)

[88] J. A. Davis, D. E. McNamara, and D. M. Cottrell, “Analysis of the fractional Hilbert transform,” Appl. Opt. 37 (29), 6911-6913 (1998).

[89] H. Ji, C. Q. Liu, "Motion blur identification from image gradients", CVPR (2008).

[90] P. A. Khaire, and N. V. Thakur, “A Fuzzy Set Approach for Edge Detection”, International Journal of Image Processing (IJIP) 6 (6), 403-412 (2012).

[91] E. Sahin, K. J. A. Ooi, C. E. Png, and D. T. H. Tan, "Large, scalable dispersion engineering using claddingmodulated Bragg gratings on a silicon chip," Applied Physics Letters 110, 161113 (2017).

[92] L. M. Lunardi, D.J. Moss, S.Chandrasekhar, L.L.Buhl, A. Hulse, P.Colbourne, G.Randall, S.Mclaughlin, "Tunable dispersion compensators based on multi-cavity all-pass etalons for 40Gb/s systems", Journal of Lightwave Technology 20, (12) 2136 (2002).

[93] A. J. Metcalf, et al., "Integrated line-by-line optical pulse shaper for high-fidelity and rapidly reconfigurable RF-filtering," Optics Express 24 (21), 23925-23940 (2016)

[94] C. Wang, et al., "Integrated lithium niobate electro-optic modulators operating at CMOS-compatible voltages," Nature 562, 101 (2018) 
[95] D. Liang, G. Roelkens, R. Baets, J. E. Bowers, "Hybrid integrated platforms for silicon photonics," Materials 3, 1782-1802 (2010)

[96] B. Stern, X. Ji, Y. Okawachi, A. L. Gaeta, M. Lipson, "Battery-operated integrated frequency comb generator," Nature 562, 401 (2018)

[97] H. Bao et al., "Laser cavity-soliton microcombs," Nature Photonics, vol. 13, no. 6, pp. 384-389 (2019).

\section{Funding:}

This work was supported by the Australian Research Council Discovery Projects Program (No. DP150104327, No. DP190101576). R. M. acknowledges support by the Natural Sciences and Engineering Research Council of Canada (NSERC) through the Strategic, Discovery and Acceleration Grants Schemes, by the MESI PSR-SIIRI Initiative in Quebec, and by the Canada Research Chair Program. Brent E. Little was supported by the Strategic Priority Research Program of the Chinese Academy of Sciences, Grant No. XDB24030000. R. M. is affiliated with 7 as an adjoint faculty.

\section{Author Contributions}

M. T., X. X. and D. J. M. developed the original concept. B. E. L. and S. T. C. designed and fabricated the integrated devices. M. T. performed the experiments. M. T., X. X., T. G. N., A. B., B. C., A. J. L., A. M. and D. J. M all contributed to the development of the experiment and to the data analysis. M. T., X. X., and D. J. M. contributed to the writing of the manuscript. D. J. M. supervised the research. 


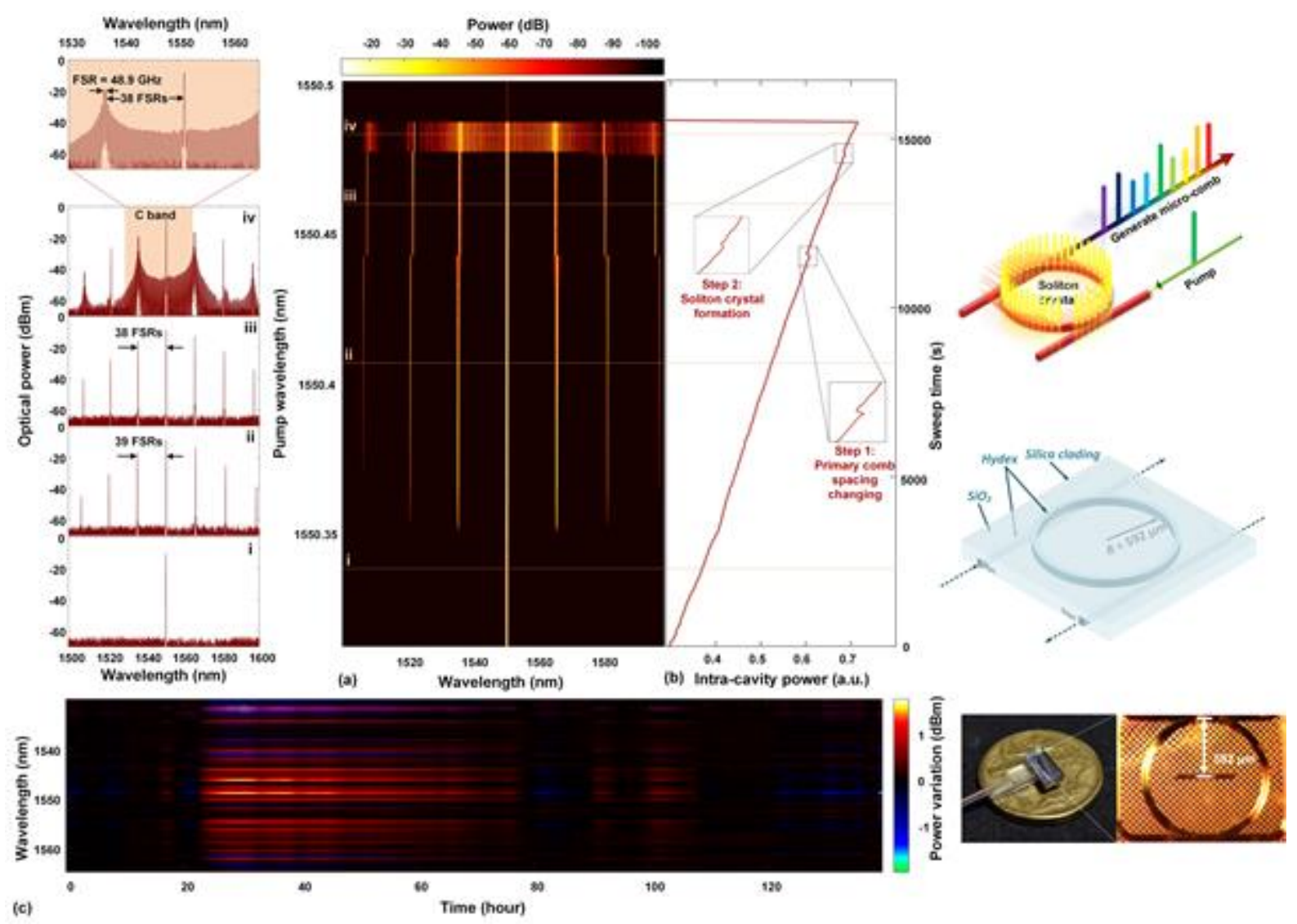

Figure 1. Schematic of soliton crystal micro-combs and generated optical spectrum. The soliton crystal is generated in a 4-port integrated micro-ring resonator (MRR) with an FSR of 49 GHz. Optical spectra of (i) Pump. (ii) Primary comb with a spacing of 39 FSRs. (iii) Primary comb with a spacing of 38 FSRs. (iv) Soliton crystal microcomb. (a) Optical spectrum of the micro-comb when sweeping the pump wavelength. (b) Measured soliton crystal step of the intra-cavity power. (c) Measured comb power stability over 140 hours (5 days). 


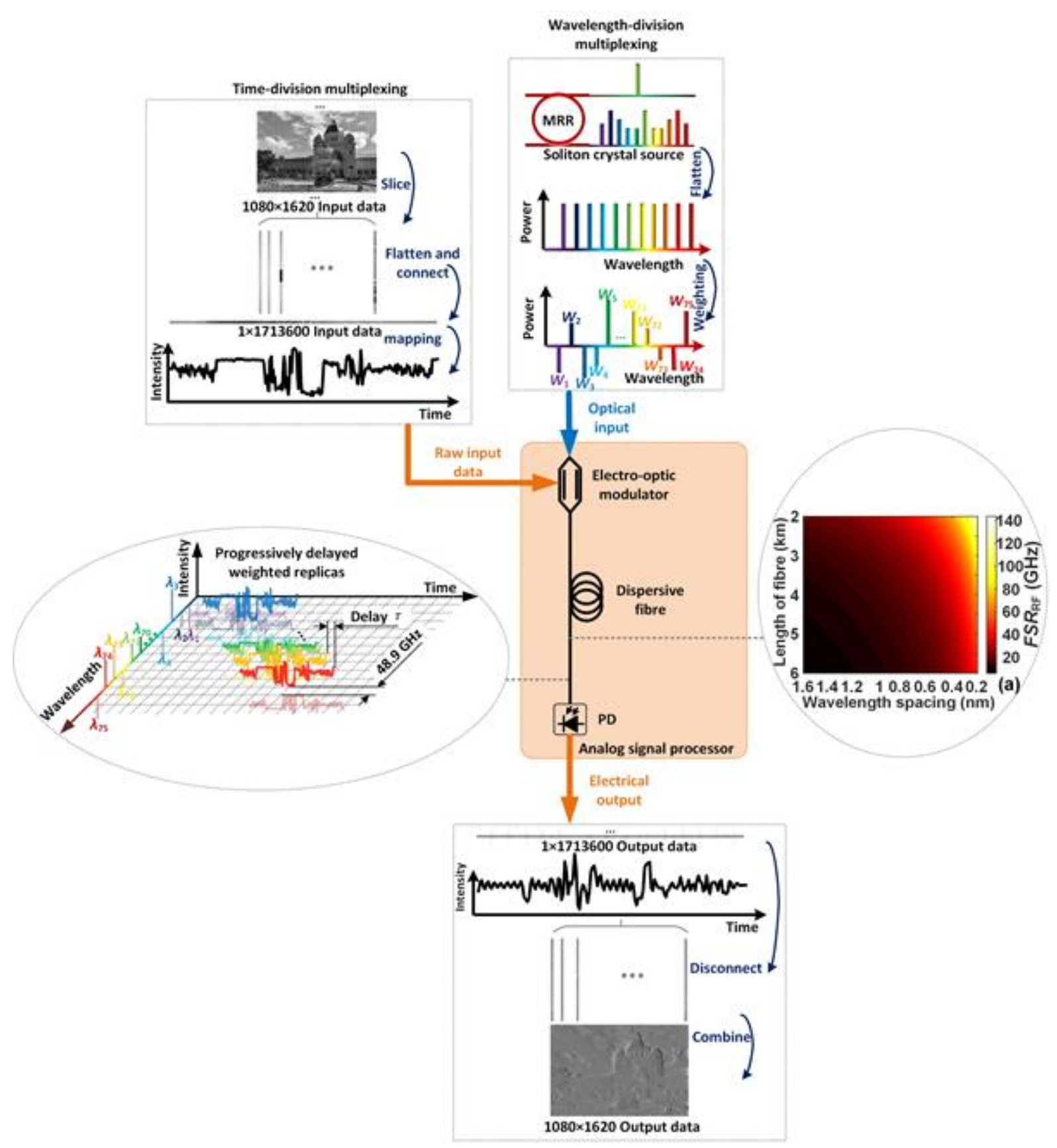

Figure 2. Operation principle of analog video processor. PD: photodetector. (a) Free spectral range of the analog signal processor according to the length of fibre and comb spacing.

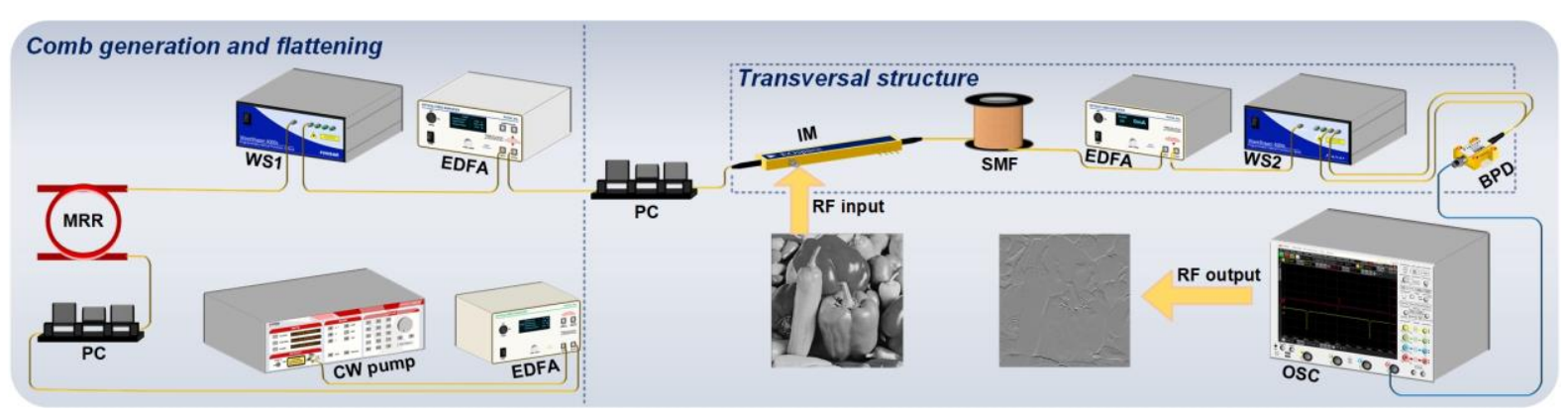

Figure 3. Experimental setup. CW pump: continues-wave pump laser. EDFA: erbium doped fibre amplifier. PC: polarization controller. MRR: micro-ring resonator. WS: WaveShaper. IM: intensity modulator. SMF: standard single mode fibre for telecommunications. BPD: balanced photodetector. OSC: high-speed oscilloscope. 


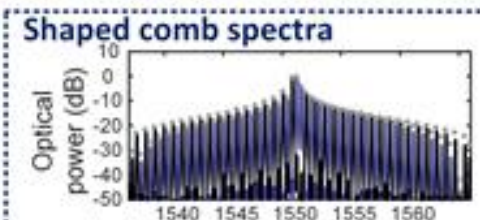

(a-i) $\begin{aligned} & 154015451550155515 \\ & \text { Wavelength }(\mathrm{nm})\end{aligned}$
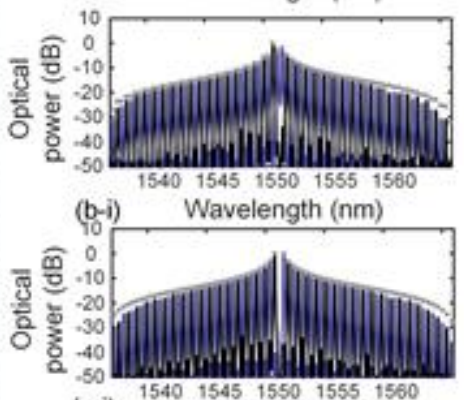

(c-i) ${ }^{1540} 1545155015551$

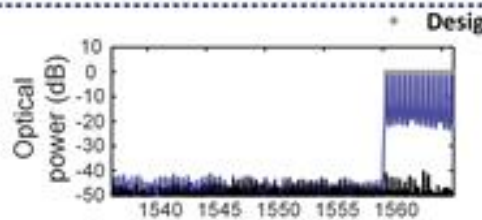

(d-i) ${ }^{1540} 1545 \quad 155015551580$

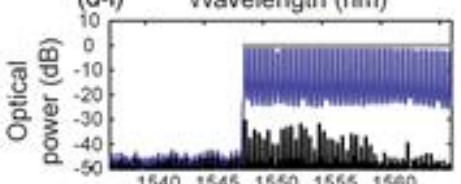

(e-i) $1540 \quad 1545 \quad 1550$ - 1555 .

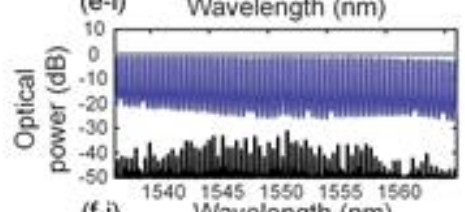

(f-i) esigned

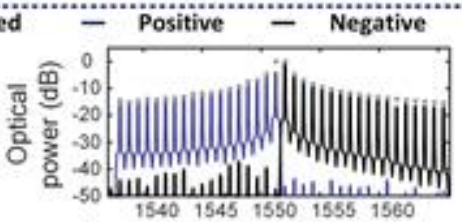

$(\mathrm{g}-\mathrm{i}) \quad$ Wavelength (nm)

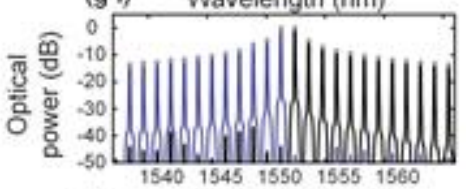

(h-i) Wavelength (nm)

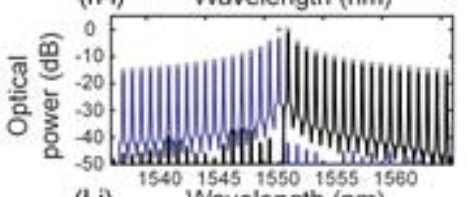

(l-i) Wavelength $(\mathrm{nm})$

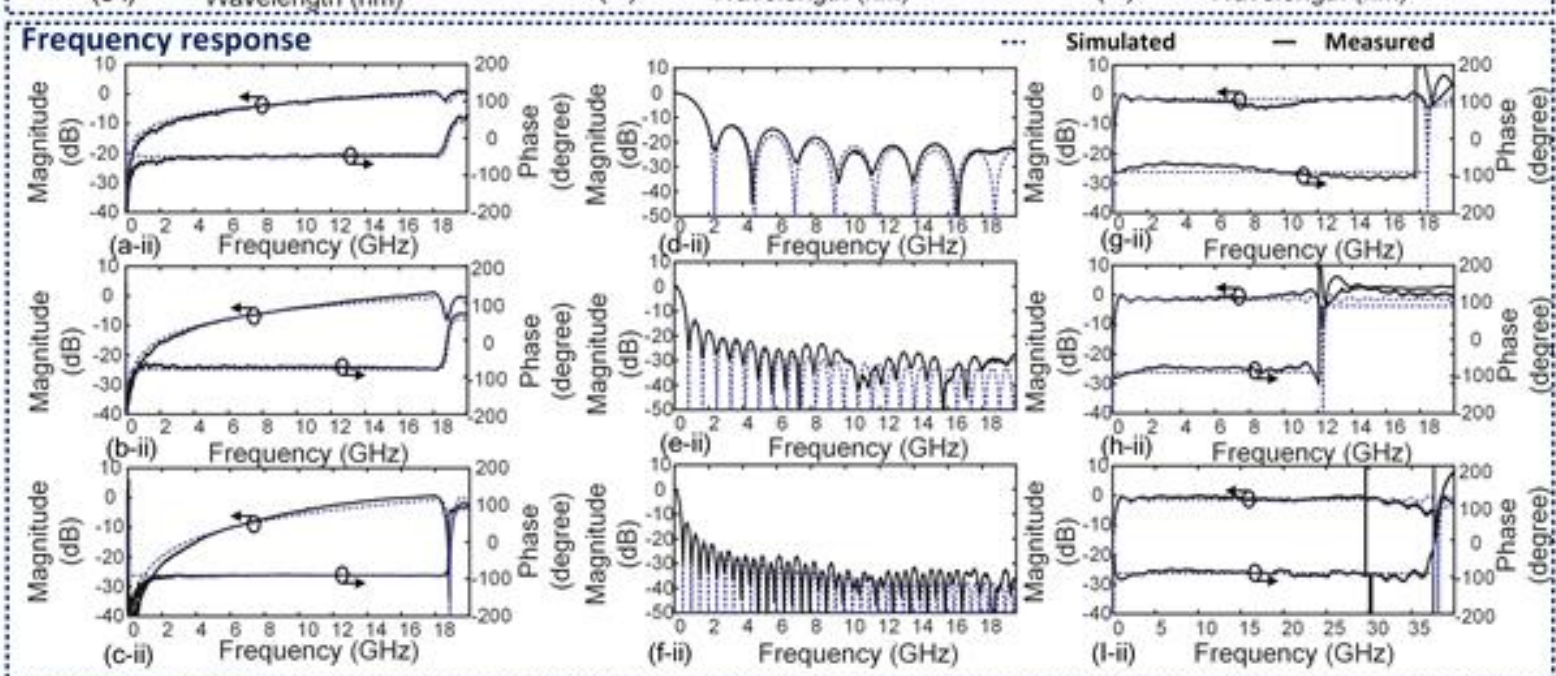

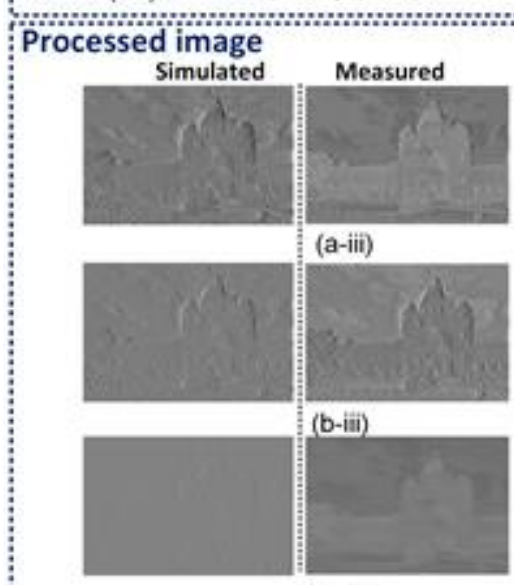

(c-iii)

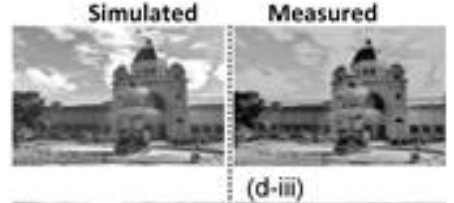

(d-iii)

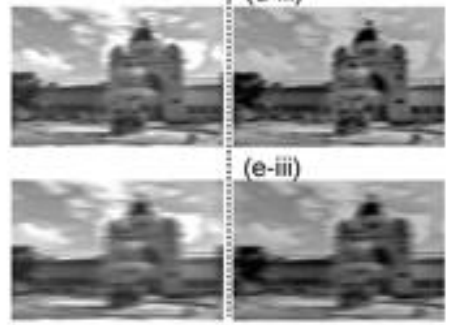

(i-iii)

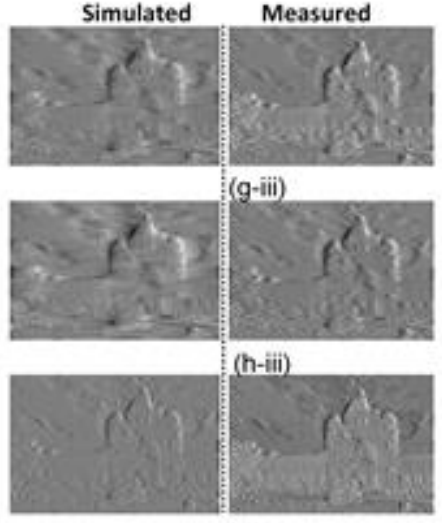

(1-iii)

Figure 4. Simulated and measured (i) shaped comb spectra, (ii) frequency response and (iii) processed high definition (HD) image of differentiator (edge detector) with fractional order of (a) 0.5 , (b) 0.75 , and (c) 1, integrator (motion blur)with tap number of (d) 15, (e) 45, and (f) 75, Hilbert transformer (edge enhancement) with operation bandwidth of (g) $18 \mathrm{GHz}$, (h) $12 \mathrm{GHz}$, and (I) $38 \mathrm{GHz}$. 
Original film
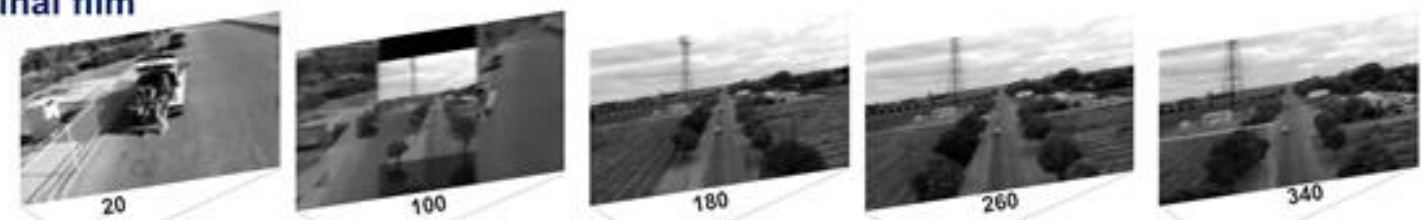

Number of frames

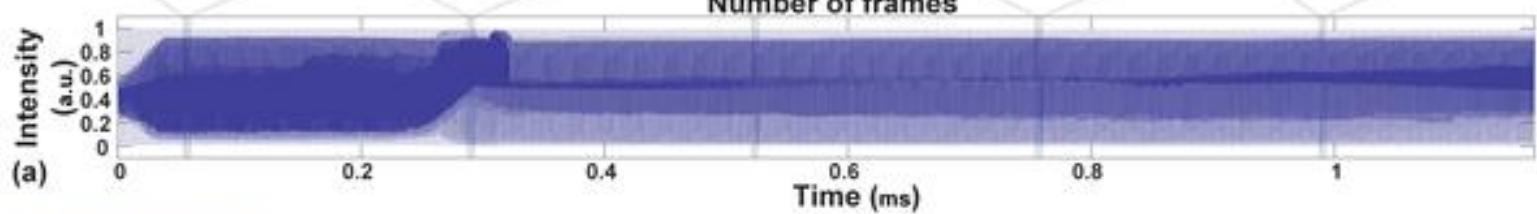

\section{Edge detection}
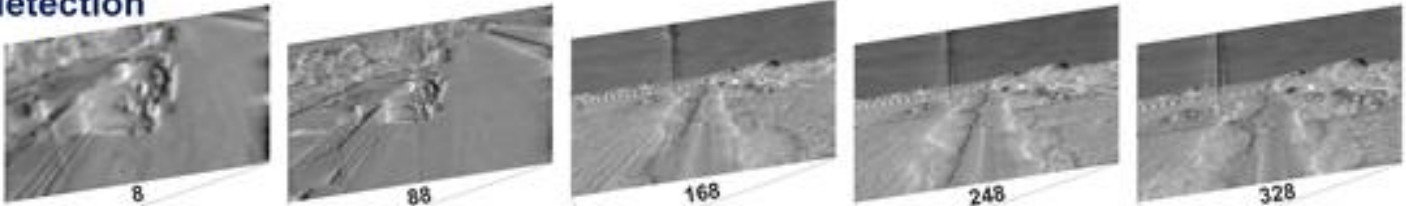

Number of frames

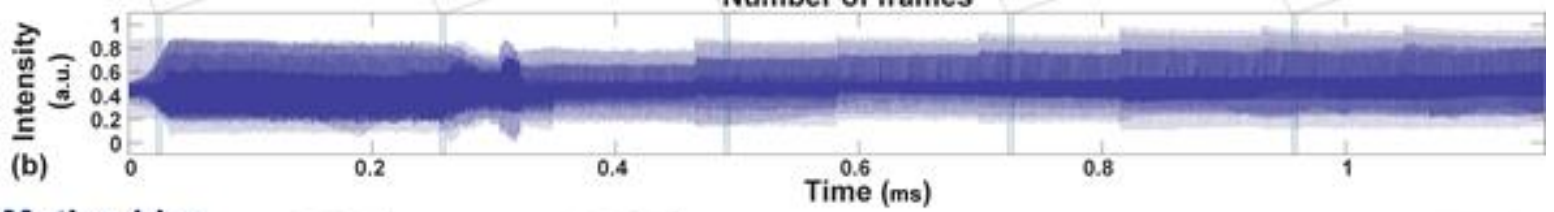

Motion blur

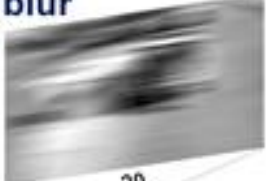

20

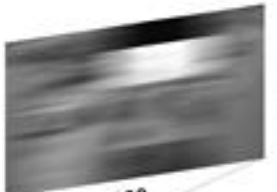

100

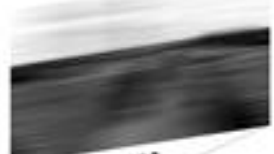

180
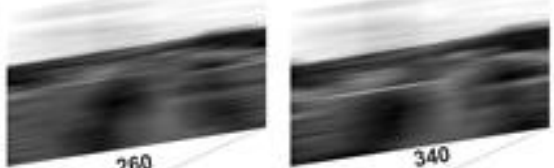

Number of frames

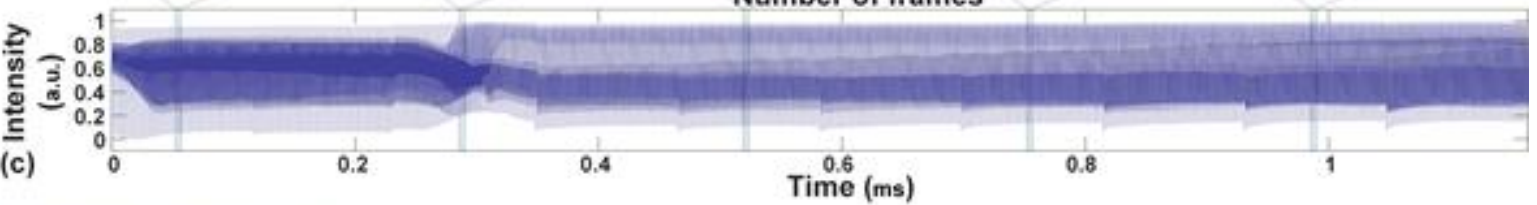

\section{Edge enhancement}
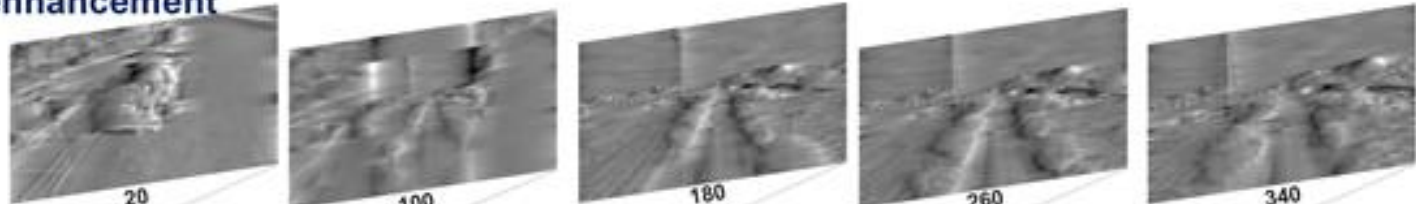

Number of frames

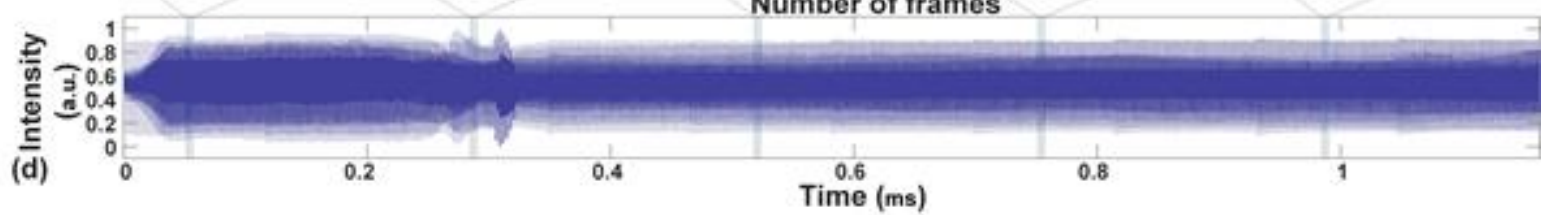

Figure 5. Measured 5 frames of the video. (a) Original video. (b) Processed video after 0.5 order differentiation. (c) Processed video after integration. (d) Processed video after Hilbert transformation with 90-degree phase shift. 


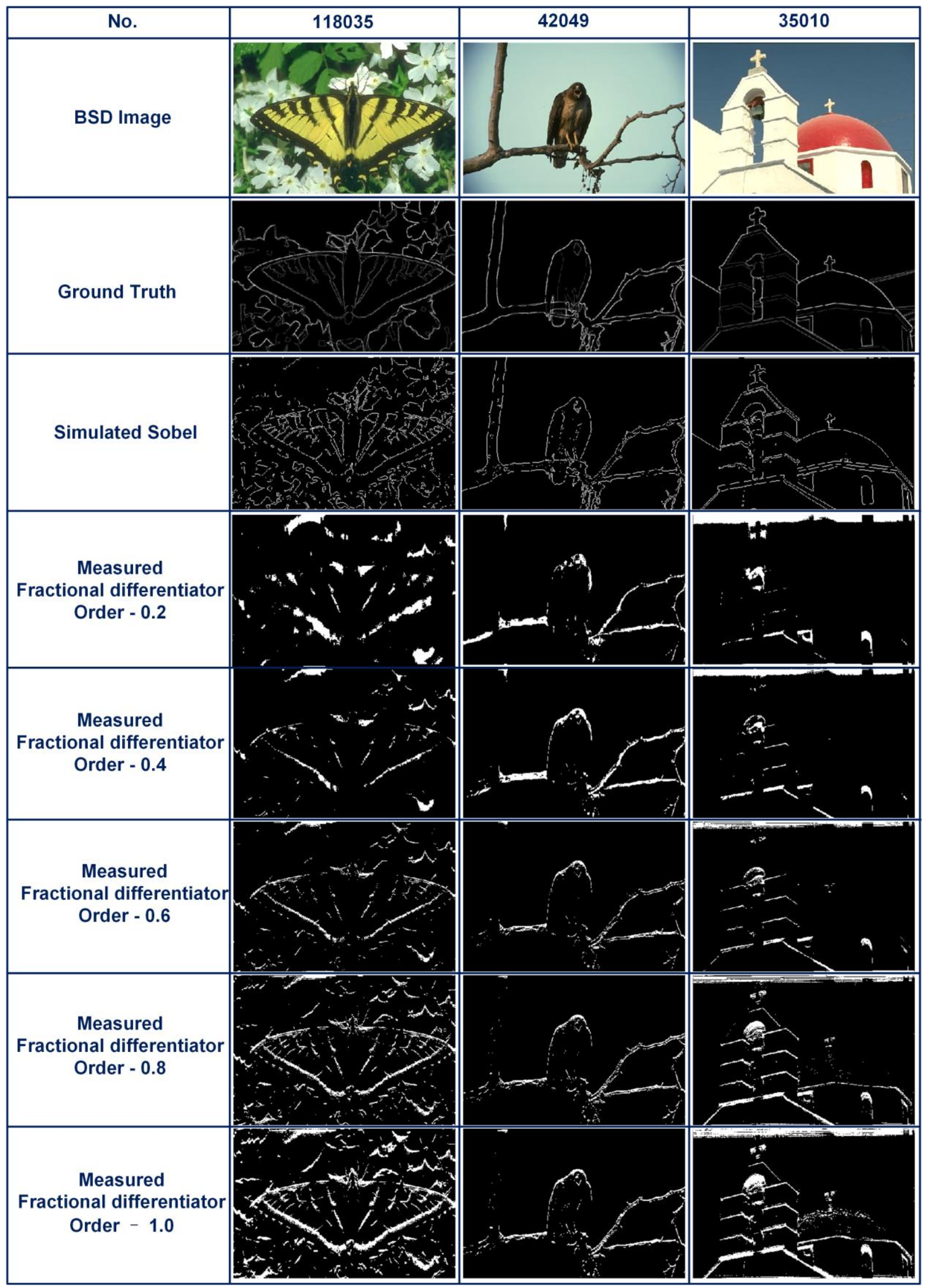

Figure 6. Ideal and measured edge detection results of BSD images with different processing approaches (Sobel algorithm and fractional differentiator with fractional order from 0.2 to 1 ).

Table 1. Comparison of calculated edge detection results of different processing approaches. 


\begin{tabular}{|c|c|c|c|c|c|c|}
\hline BSD image No. & \multicolumn{2}{|c|}{118035} & \multicolumn{2}{c|}{42049} & \multicolumn{2}{c|}{35010} \\
\hline $\begin{array}{c}\text { Simulated } \\
\text { Sobel }\end{array}$ & 12.2488 & 0.0068996 & 15.8362 & 0.0049214 & 11.3226 & 0.011988 \\
\hline $\begin{array}{c}\text { Measured fractional } \\
\text { differentiator order - 0.2 }\end{array}$ & 6.2279 & 0.010047 & 12.1913 & 0.016736 & 6.079 & 0.0092251 \\
\hline $\begin{array}{c}\text { Measured fractional } \\
\text { differentiator order - 0.4 }\end{array}$ & 4.3626 & $\mathbf{0 . 0 0 7 9 9 0 7}$ & 12.8774 & 0.020768 & 4.7099 & 0.0066706 \\
\hline $\begin{array}{c}\text { Measured fractional } \\
\text { differentiator order - 0.6 }\end{array}$ & 7.7731 & $\mathbf{0 . 0 0 6 3 8 2 9}$ & 10.1273 & 0.011288 & 6.3306 & 0.0078077 \\
\hline $\begin{array}{c}\text { Measured fractional } \\
\text { differentiator order - 0.8 }\end{array}$ & 12.5839 & 0.012145 & 11.2812 & 0.013869 & 11.1173 & 0.014635 \\
\hline $\begin{array}{c}\text { Measured fractional } \\
\text { differentiator order - 1.0 }\end{array}$ & 14.614 & 0.021003 & 10.1897 & 0.015944 & 11.8308 & 0.017894 \\
\hline
\end{tabular}




\section{Methods}

To achieve the designed tap weights, the generated soliton crystal microcomb was shaped in power using liquid crystal on silicon based spectral shapers (Finisar WaveShaper 4000S). We used two WaveShapers in the experiments - the first was used to flatten the microcomb spectrum while the precise comb power shaping required to imprint the tap weights was performed by the second, located just before the balanced photo detector (Finisar BPDV2150R). The negative tap weights were achieved by separating the wavelength into two spatial outputs of the WaveShaper according to the tap weights and then detected by the balanced photo detector. A feedback loop was employed to improve the accuracy of comb shaping, where the error signal was generated by first measuring the impulse response of the system with a Gaussian pulse input and comparing it with the ideal channel weights. (The shaped impulse responses for the video processor are shown in the Supplementary Materials).

The electrical input data was temporally encoded by an arbitrary waveform generator (Keysight M8195A, 65 Giga Symbols/s, $25 \mathrm{GHz}$ analog bandwidth), the raw input matrix is first sliced horizontally or vertically into multiple rows or columns, they were then flattened into vectors and connected head-to-tail to form the desired vector. The detailed flattening process for the video processing is shown as in Fig. 7. The desired vector was then multicast onto the wavelength channels via a $40 \mathrm{GHz}$ intensity modulator (iXblue). For the high definition image with a resolution of $1080 \times 1620$ pixels and video with a frame rate of 30 frames per second processing, we used sample points at a rate of 54 Giga samples/s to form the input symbols. We then employed a $3.96 \mathrm{~km}$ length of dispersive fibre that provided a progressive delay of $27 \mathrm{ps} / \mathrm{channel}$, the generated electronic waveforms for the images are shown in the Supplementary Materials. Finally, the electrical output waveform was resampled and digitized by a high-speed oscilloscope (Keysight DSOZ504A, 80 Giga Symbols/s) to extract the final output. We further characterized the transmission response using a vector network analyser (Agilent MS4644B $40 \mathrm{GHz}$ bandwidth).

There are a number of factors that can lead to tap errors during the comb shaping, thus leading to nonideal frequency response of the system as well as deviations between the experimental results and theory. These factors mainly include the instability of the optical micro-combs, the accuracy of the WaveShapers, the gain variation with wavelength of the optical amplifier, the chirp induced by the optical modulator, the second-order dispersion induced power fading, and the third-order dispersion of the dispersive fibre. To combat these, real-time feedback control paths can be employed to effectively reduce the errors of the accuracy of WaveShapers and the gain variation with wavelength of the optical amplifier, in this approach, replicas of an RF Gaussian pulse are measured at all wavelengths to obtain the impulse response of the system, whose peak intensities are further extracted to obtain accurate RF-to-RF wavelength channel weights. Following this, the extracted channel weights are subtracted from the desired weights to obtain an error signal that is used to program the loss of the WaveShaper. After several iterations of the comb shaping loop, an accurate impulse response that compensates the non-ideal impulse response of the system can be obtained, thus significantly improving the accuracy of the photonic based analog image/video processing. 


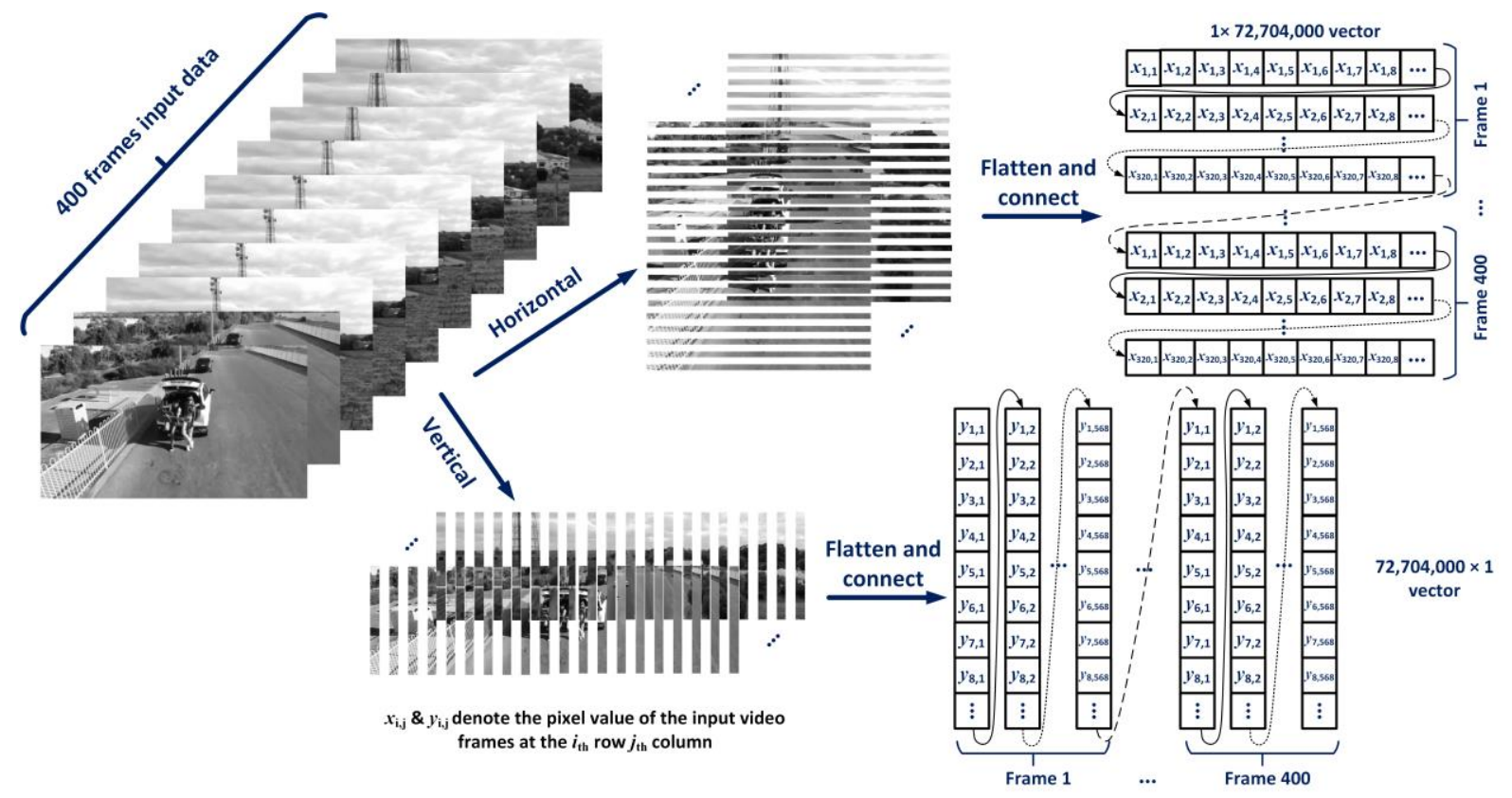

Figure 7. Diagram illustrating the flattening method applied to the input video frames. 